Michael, K. D., Huelsman, T. J. , \& Crowley, S. L. (2005). Interventions for child and adolescent depression: Do professional therapists produce better results? Journal of Child and Family Studies, 14:2, 223-236. June 2005 (ISSN: 1573-2843) Version of record published by Springer Verlag DOI: 10.1007/s10826-005-5050-8

\title{
Interventions for Child and Adolescent Depression: Do Professional Therapists Produce Better Results?
}

\author{
Kurt D. Michael, Timothy J. Huelsman, and Susan L. Crowley
}

\begin{abstract}
We reviewed and analyzed child and adolescent depression treatment studies (1980-2001) through a comprehensive literature search. The outcome data from 19 studies (31 treatments) were extracted and weighted standard mean effect sizes were computed. Outcomes were compared across two levels of therapist training: professional and graduate student. Moreover, age was examined to test for differential effects on treatment outcome. Overall, professionals and graduate student therapists produced impressive yet commensurate outcomes when treating depressed youth. There were no significant differences found when treating children versus adolescents. The implications and limitations are reviewed, as are the suggestions for future research.
\end{abstract}

\footnotetext{
ARTICLE

The importance of therapist characteristics and their impact on treatment outcome has been well documented (e.g., Beutler, Machado, \& Allstetter Neufeldt, 1994; Elkin, 1999). We now have a substantial body of literature that strongly supports the therapeutic benefits of the so-called "common factors," first described by Carl Rogers, which include constructs such as empathy, warmth, and genuineness (Patterson, 1984). Moreover, when working with children and adolescents in particular, it now appears that clients who have a negative view of their therapists are far more likely to drop out of therapy prematurely (Garcia \& Weisz, 2002). Other therapist variables, including level of training and years of experience, have also been examined to determine their potential contribution to treatment outcome (e.g., Kolko, Brent, Baugher, Bridge, \& Birmaher, 2000). In order to determine whether an association exists between the level of training and
} 
outcome, researchers have often selected a sample of studies (e.g., professionals versus paraprofessionals), calculated an overall effect size (ES) for each study, and coded differential levels of training across the various studies. However, the findings from the majority of these studies are not only equivocal, but also unsettling given that a number of studies support the assertion that paraprofessionals and/or lesser trained practitioners are equally effective and, in some cases, more effective than their more highly trained colleagues (e.g., Berman \& Norton, 1985; Stein \& Lambert, 1984; Durlak, 1979; Hattie, Sharpley, \& Rogers, 1984).

In a review of related literature, Christensen and Jacobson (1994) suggested poignantly, "years of study and training should dramatically alter a person's ability to conduct professional work. In most professions, it would be ludicrous to compare a trained and an untrained person. It is hard to imagine a study comparing trained and untrained surgeons, or trained and untrained electricians for that matter" (p. 9). Indeed, the very notion that lesser trained clinicians produce equivalent or superior outcomes probably strikes fear in the hearts of clinical training directors everywhere. If spending substantial amounts of time, money, and energy to become more "expert" in the field of psychotherapy does not translate into appreciable benefits for clients and society at large, it begs the question, why bother? Alternatively, it might be true that a professional psychotherapist provides a significant and worthwhile service to those in need well above and beyond what a layperson or lesser trained individual can produce, yet we are unable to substantiate these claims of enhanced effectiveness in an empirically responsible way given confounds and limitations in the data.

Stein and Lambert (1995) pointed out that in most studies where the relationship between training level and outcome was examined, this issue was not the primary hypothesis and so clear delineations between various levels of training were not typically conducted. To complicate matters, analyzing potential interactions between level of training and outcome at a broad level (e.g., psychotherapy overall) is even more difficult given the multiple layers of confounds between variables. Therefore, in order to systematically chip away at the question, it might be useful to examine a narrower sample of studies by problem type (e.g., depression treatment studies) or by client age group (e.g., child and adolescent versus adult) to reduce the number of potential confounds.

Whether the provocative findings outlined above are attributable to actual negligible effects of training or to methodological problems, this issue is of considerable import given the enormous financial and practical costs of pursuing graduate training in psychotherapy. Further, insurance companies would seemingly be interested in the question, especially if lesser trained (and presumably less expensive) therapists could adequately deliver the treatments. While it is well beyond the scope of this study to examine the overall relationship between level of training and treatment outcome across multiple problem types, the primary purpose of this investigation is to evaluate whether professional therapists produce better results than lesser trained therapists when treating depressed children and 
adolescents.

The overall effectiveness of psychotherapy with depressed children and adolescents has been supported in at least three recent meta-analytic reviews, but it remains less clear whether there is evidence of differential effectiveness when the level of therapist training is taken into account (Michael \& Crowley, 2002; Reinecke, Ryan, \& DuBois, 1998;Weisz,Weiss, Han, Granger, \& Morton, 1995). In a meta-analysis of 108 controlled studies conducted by Weisz et al. (1987), the main effect of therapist training and outcome was not statistically significant ( $p=.43$ ) across three levels of training, including professionals, graduate students, and paraprofessionals. Weisz et al. (1987) provided the following definition of the three levels of training "(a) professionals who held a doctor's or master's degree in psychology, education, or social work; (b) graduate students who were working towards advanced degrees in psychology, education, or social work; and (c) paraprofessionals who were parents, teachers, or others lacking mental-healthrelated graduate training but trained to administer the therapy" (p. 545).

Weisz et al. (1987) reported that there was no evidence of differential success across three levels of training (i.e., paraprofessionals, graduate students, professionals) for "undercontrolled" problems (e.g., delinquency, hyperactivity, impulsivity, now more commonly referred to as externalizing problems; see Achenbach \&McConaughy, 1992 for further review). However, for youngsters who presented with "overcontrolled" problems (e.g., phobias, anxiety, social withdrawal, now more commonly referred to as internalizing symptoms; see Reynolds, 1990 for further review), treatment effectiveness was positively associated with increased training. Nonetheless, given that none of studies were explicitly coded as "depression" treatments, it was not possible to discern whether increased training was associated with better outcomes in studies targeting depressed youth.

In a more recent meta-analysis of 150 additional controlled child and adolescent outcome studies, Weisz et al. (1995) reported that overall, paraprofessionals produced better results than professionals and graduate students across all problem types. However, when treating internalizing problems there was a statistically significant interaction wherein professionals produced superior outcomes when compared to their less trained counterparts (i.e., professionals > graduate students $>$ paraprofessionals). These findings were reversed when treating externalizing problems (i.e., paraprofessionals > professionals = graduate students; the professionals and graduate students did not differ reliably according to the authors). Taken Together, these data (Weisz et al., 1987, 1995) suggest that there is an intriguing interaction between professional training and problem type: therapists with more training appear to produce better results with youth who present with internalizing problems, broadly subsuming clinical conditions such as phobias/anxiety, social withdrawal, isolation, depression, and somatic complaints. Conversely, when it comes to treating externalizing problems, paraprofessionals appear to have the edge. Nonetheless, it remains to be demonstrated whether the relationship between training and problem type is evident in a more homogeneous 
and up-to-date sample of treatment trials designed to address child and adolescent depression in particular.

In a recent clinical trial for adolescent depression, Kolko et al. (2000) examined whether therapist background (i.e., years of experience) moderated treatment outcome across 3 treatments (cognitive-behavioral therapy, systematic-behavioral family therapy, and nondirective supportive therapy). The authors reported that there were no statistically significant interactions between therapist background and outcome. However, in discussing these null findings, Kolko et al. (2000) suggested, "most treatment studies with depressed youths have not evaluated therapist effects or the impact of therapist characteristics on outcome" (p. 661). Moreover, other researchers have lamented the fact that there is a paucity of studies that attempt to address the interaction between therapist variables and treatment outcome (e.g., Reinecke et al., 1998). Thus, it appears that there is ample justification to investigate whether particular therapist variables such as training level are differentially related to outcome when treating depressed youth.

In sum, given the equivocal nature of the findings regarding level of training and outcome, and the apparent differential effectiveness when treating internalizing and externalizing problems, examinations of a narrower scope are justified in order to reduce the number of potential confounds and correlates. Moreover, given the previous findings that suggest lesser trained clinicians produce commensurate and in some cases, superior results, it was important to address the question as to whether professional training enhances one's ability to conduct psychotherapy. Thus, the primary question addressed in the present study was whether professional training was associated with better outcomes in a more homogeneous and current sample of child and adolescent depression treatment studies. As mentioned previously, in the other meta-analytic studies that reported an interaction between level of training and problem type (i.e., Weisz et al., 1987, 1995), the treatment trials reviewed were not described as depression treatments (Weisz et al., 1987) or only six published depression treatments were included (Weisz et al., 1995). In addition to including the six published studies reviewed by Weisz et al. (Butler, Miezitis, Friedman,\&Cole, 1980; Kahn, Kehle, Jenson,\&Clark, 1990; Lewinsohn, Clarke, Hops, \& Andrews, 1990; Liddle \& Spence, 1990; Reynolds \& Coats, 1986; Stark, Reynolds, \& Kaslow, 1987), another thirteen studies were included in the present meta-analysis to provide an updated picture of the possible interaction between level of training and problem type (i.e., depression).

A secondary question addressed in the present study was whether age of the child influenced outcome given the findings from some recent meta-analytic reviews. For example, Weisz et al. (1995) reported that across 150 child and adolescent trials "treatment outcomes were better for adolescents than for children" (p. 461). Further, Michael and Crowley (2002) reported that when treating depressed youth in particular, the mean effect size for adolescent samples was higher than the mean ES for child samples, regardless of experimental design (i.e., pre/post vs. controlled). However, in contrast to these 2 studies, Weisz et al. 
(1987) reported "therapy proved more effective for children (ages 4-12) than for adolescents (ages 13-18)" (p. 542). Thus, the findings regarding an interaction between age and outcome are equivocal. So, the potential interaction between level of training, age, and outcome was examined in the present study. For purposes of clarity and consistency with previous studies, children were referred to as those who were between 6 and 12 years-old, whereas adolescents were identified as those who were between the ages of 13 and 18 .

\section{METHOD}

\section{Population and Sample}

The population for this investigation included empirical studies on the treatment of child and adolescent depression. The overall sample for this investigation included 19 psychosocial treatment studies targeting child and adolescent depression published between 1980 (the first published controlled study on the treatment of youth depression) and 2001. There were 31 separate treatments across the 19 studies. Case reports and single-subject designs were not included. Of the 19 studies, 12 were conducted within the context of a between-subject design with wait-list $(N=7)$, no-treatment $(N=4)$, or clinical monitoring $(N=1)$ control conditions. Further, 2 of the studies (Butler, Miezitis, Friedman, \& Cole, 1980; Liddle \& Spence, 1990) included an attention-placebo condition in addition to one of the control conditions described above. For a between-group study to be considered a controlled trial, we required random assignment to one of the aforementioned control conditions. Further, among the controlled studies included in the analysis, random assignment appeared to be rigorous and not simply based on convenience (e.g., assigned to a particular condition due to scheduling constraints). Across the 12 controlled studies, there were a total of 18 active treatments. The remaining 7 studies were evaluated within the context of a pre/post design. Across the 7 pre/post studies, there were a total of 13 active treatments.

The articles were located through a comprehensive search strategy including an extensive computer search of databases such as PsycINFO, ERIC, and Medline. Further, in an effort to be as comprehensive as possible, manual searches of the reference lists from the obtained articles were conducted in an effort to find additional articles and several authors active in this area of investigation were contacted to inquire about "in press" studies or unpublished manuscripts. The strategy was employed in an effort control for the "file drawer effect" (Rosenthal, 1979), or the possibility of omitting relevant studies (e.g., unpublished manuscripts, dissertations) that might help to inform the research question at hand. The one unpublished study located and included in the analyses (Curry \& Wells, 1998) has since been published (Curry, Wells, Lochman, Craighead, \& Nagy, 2003). Manual searches of a variety of peer-reviewed journals were completed as well. The journals selected to manually search was based on 3 criteria: (1) whether the journal had ever published a depression treatment trial; (2) whether the journal had ever published 
a child/adolescent treatment trial; or (3) whether the journal focused on child/adolescent psychopathology and/or depression in particular. The journals that were manually searched included the Journal of Consulting and Clinical Psychology, the Journal of the American Academy of Child and Adolescent Psychiatry, Behavior Therapy, Archives of General Psychiatry, School Psychology Review, Adolescence, Behavioral Psychotherapy, the Journal of Abnormal Child Psychology, the Journal of Clinical Child Psychology, the American Education Research Journal, and the Journal of Affective Disorders.

Finally, a comprehensive search of unpublished theses and dissertations via ProQuest Dissertation Abstracts was completed dating back to 1980. A total of 10 potentially relevant dissertations were located, all of which were ordered. Four of the dissertations ordered were not received due to restrictive lending policies at the institutions. Of the six dissertations received, one was appropriate for coding (Hickman, 1994) and included in the final sample of studies.

For a study to be included in the investigation, the effects of a psychosocial treatment on child and adolescent depression had to be examined. The following specific criteria must have been met as well: the study had to be a within- or between-subjects group design; the sample was targeted for intervention based upon presenting depressive symptomatology or a depressive diagnosis; the subjects targeted for intervention were between the ages of 5-18; the treatment was psychosocial in nature (e.g., group, individual, family); and at least one depression outcome measure was administered once the intervention was completed.

\section{Design and Analysis}

A meta-analytic design was used during the course of this study, whereby the results from related treatment studies are compared (Glass, 1977). In the present study, a standardized mean difference weighted effect size was calculated (following the Hedges \& Olkin, 1985, technique for correcting Cohen's $d$ for sample size). A coding sheet was developed so that each of the treatment outcome studies was evaluated on the identified variables (i.e., level of training, age). All treatment studies were double-coded by the primary researcher and a Ph.D. candidate in clinical psychology that underwent extensive training in specific coding procedures. Inter-rater agreement was calculated by using Cohen's Kappa (Cohen, 1960). The Kappa coefficient was .84 for the level of therapist training and .96 for the age. Disagreements in the coding of any variables were reconciled through consultation and clarification between the coders.

The outcomes from the treatment studies were analyzed by computing effect sizes. The calculation of effect sizes for the studies was based upon measures of depressive symptoms and depressive diagnoses (i.e., self-report measures, diagnostic interviews) at posttreatment. In some cases, the investigators used more than one self-report measure or combined self-report data with interview findings. 
Of the 19 psychosocial studies, 6 used a single self-report measure to assess outcome, while 3 studies combined 2 self-reportmeasures to determine outcome. The remaining 10 studies used a combination of self-report measures and interview data to assess outcome. For studies in which more than one assessment device was included, the measures were collapsed to yield one overall effect size for each treatment in each study to avoid the potential limitation of unequal weighting of studies (Glass, 1977).

Effect sizes were calculated by the ES computer software program (Johnson, 1989) based upon the outcome data (i.e., means, standard deviations, sample sizes). In cases where the means and standard deviations were not provided, effect sizes were computed from other data reported in the studies (e.g., $F$-ratios or $t$ statistics). Given that direct comparisons of between-and within-group effect sizes are inappropriate in the same meta-analysis, pre/post effect sizes were calculated within and across all studies to facilitate comparisons regardless of methodological design and to increase power. The procedure of calculated pre/post effect sizes across design types has been described extensively (Becker, 1988; Lipsey \& Wilson, 2001; Rosenthal, 1994) and used for similar purposes in at least three recent meta-analytic studies (DuPaul \& Eckert, 1997; Michael \& Crowley, 2002; Wilson, Lipsey, \& Derzon, 2003).

To code for the level of training, two categories (i.e., professionals, graduate students) were created. These two categories were a replication of the coding system first described and utilized by Weisz et al. (1987) wherein professionals, graduate students, and paraprofessionals were included. However, only two categories were used in light of the fact that only two paraprofessional treatment studies were located in the literature review. As a result, both of the studies and the paraprofessional classification were subsequently dropped from the analyses due to an insufficient number of observations in this category. Professionals were defined as individuals holding an advanced degree (i.e., doctoral degree or a terminal master's degree) with specific training in psychology, social work, psychiatry, or education. Graduate students were defined as individuals working towards professional or advanced degrees in psychology, social work, psychiatry, or education. If the level of therapist training reported within a particular study was vague (i.e., undefined mixture of graduate student and professional therapists) or if the level of training was not explicitly described or coded (e.g., Clarke, Rohde, Lewinsohn, Hops, \& Seeley, 1999), it was excluded from the analysis.

Of the 19 studies reviewed, 9 studies were coded as "professional" studies (16 active treatments). Of the 9 professional studies, 3 had only doctoral-level therapists (either $\mathrm{PhD}$ or $\mathrm{MD}$ ); 5 had a combination of doctoral and master's level therapists (range of post-degree clinical experience: 3-20 years); and 1 had only master's level therapists (median clinical experience: 10 years). One of the professional studies (Santor \& Kusumakar, 2001) included 2 advanced clinical psychology $\mathrm{PhD}$ students (both with a master's degree) among its 9 professional therapists. A decision was made to code this study as "professional" given that a 
clear majority (78\%) of therapists were described as professionals with terminal degrees and who possessed a minimum of 3 years of clinical experience.

The remaining 10 trials reviewed were coded as "graduate student" studies (15 active treatments). Although the therapists' status as a graduate student therapist was made explicit in each of the studies, their standing as a doctoral-level student was made clear in only 4 studies. In the remaining 6 studies, the words "advanced graduate student," "trainee," or "second year clinical psychology trainee" were used to indicate their level of training. Further, across these 6 studies, it was not made explicit whether the graduate students possessed a master's degree nor was the years of clinical experience evident. One of the graduate student studies (Weisz, Thurber, Sweeney, Proffitt, \& LeGagnoux, 1997) included 1 licensed faculty member among its 6 therapists, 5 of which were explicitly described as doctoral-level graduate students. A decision was made to code this study as "graduate student" given that a clear majority (83\%) of therapists were described as "doctoral level students in clinical psychology, all having clinical training and supervision before this study" (p. 704). Overall, the distinctions between professionals and graduate students across the 19 studies were based upon the actual descriptors of the therapists and in many cases, the references made to "terminal" degrees and years of clinical experience (mentioned explicitly in 6 professional studies).

In terms of coding age, the procedure described by Weisz et al. (1995) was replicated: "child" studies were defined as having a mean age of below 13 years old whereas "adolescent" studies were defined as having a mean age of equal to or greater than 13 years-old. There were 12 active "child" treatments and 19 active "adolescent" treatments.

\section{RESULTS}

\section{Descriptive Statistics}

The psychosocial studies were published or conducted between 1980 and 2001 , with the vast majority (85\%) of studies published after 1990. Across all studies, there were a total of 885 participants between the ages of 7 and 18 years old. The mean age of participants across all studies was 13.7 years old, with the age of participants ranging from 9.2 to 16.5 years old. The number of participants in each study ranged from 9 to 107 (median $=48$ ). The average percentage of female subjects across all 19 studies was approximately $58 \%$. However, only 2 of the 19 studies (11\%) reported separate findings based on sex.

There were 31 active treatments across all studies. The modal treatment regimen was cognitive-behavioral therapy (group or individual; $n=15$ ), followed 
by interpersonal therapy $(n=4)$, non-directive, supportive individual therapy $(n=3)$, social skills group therapy $(n=3)$, and relaxation group therapy $(n=3)$, behavioral problem-solving $(n=1)$, structured behavior family therapy $(n=1)$, and role-play $(n=1)$. All 31 treatments were based upon clinically significant depressive symptoms $(45 \%)$ or one or more depressive diagnoses (55\%; diagnosis of Major Depressive Disorder and/or Dysthymic Disorder). Of the 19 studies included in the review, 16 explicitly referred to the treatment as a manualized protocol, whereas the treatments in 3 studies were not described as "manualized." The number of sessions in across studies ranged from 6 to 36 (median $=12$ ) and the number of weeks for each treatment ranged from 5 to 14 weeks (median $=8$ ).

\section{Outcome by Level of Training and Age}

The mean pre/post effect size across all 31 treatments reviewed in the present study was 1.47 , far exceeding the conventional threshold for a "large" effect (.80; Cohen, 1988). Both professional therapists and graduate student therapists produced rather large treatment effects that were significantly different from zero (using a random effects model; see $95 \%$ confidence intervals, Table I). However, the difference in effect size between professional therapists and graduate students was not statistically significant $(t 29=-.71, p=.48, r=.131)$. Similarly, when age was taken into account, both groups were associated with large effect sizes

Table I. Means, Standard Deviations, Number of Treatments and 95\% Confidence Intervals for Each Study Condition

\begin{tabular}{lcccc}
\hline \multicolumn{1}{c}{ Variable/levels } & Mean ES & $S D$ & Nof treatments & $95 \% C I$ \\
\hline $\begin{array}{l}\text { Level of training } \\
\quad \text { Graduate student }\end{array}$ & 1.38 & .56 & 15 & $1.07-1.69$ \\
$\quad$ Professional & 1.56 & .80 & 16 & $1.13-1.98$ \\
Age group & & & & \\
$\quad$ Children & 1.50 & .55 & 12 & $1.15-1.85$ \\
$\quad$ Adolescents & 1.45 & .77 & 19 & $1.08-1.82$ \\
\hline
\end{tabular}

Note. There were no interactions found for effect size and type of intervention (cognitive-behavioral vs. interpersonal) due to an insufficient clarity of the treatment descriptions. The effect size estimates between interventions based on a diagnosis (mean $E S=1.61, S D=.54$ ) versus elevated symptoms (mean $E S=$ $1.37, S D=.77)$ were not significantly different $\left(t_{(29)}=.95, p=.44\right)$.

(see Table I), but there was no difference between the groups $(t 29=.20, p=.83$, $r=.037)$. 


\section{DISCUSSION}

Based upon the results obtained in this study, it does not appear that professional therapists produce better outcomes when treating children and adolescents with depression. These data are not consistent with the findings from at least two previous and large meta-analytic reviews involving children and adolescents (Weisz et al., 1987, 1995) which indicate that professionals produce better outcomes when treating internalizing problems in children and adolescents. This suggests that while professional therapists appear to have the edge when treating a broadly-defined rubric of internalizing disorders, the differential effects essentially disappear when depression interventions are considered alone.

Despite the divergence of these data with some of the findings from large child and adolescent meta-analytic reviews (Weisz et al., 1987; 1995), in two recent studies involving depressed adolescents, researchers reported non-significant interactions based upon other relevant therapist variables (e.g., specialized versus non-specialized background, years of experience). In an open trial of 25 adolescents with moderate to severe depression, Santor and Kusumaker (2001) analyzed the effectiveness of therapists with more "specialized" psychotherapy backgrounds (i.e., defined as doctoral psychologists, psychiatric residents) versus therapists with less specialized backgrounds (i.e., clinical nurses, social workers). Their analyses did not reveal any statistically significant differences between groups on any of several outcome measures. In another recent trial of 103 depressed adolescents, Kolko et al. (2000) reported that therapist experience (in years) did not interact with outcome across any of the dependent measures. Thus, in more circumscribed investigations of depressed youth (including the present study), more or increasingly specialized training does not appear to be related to better outcomes for the children and adolescents.

We offer some possible interpretations of these equivocal findings between the 2 cited large meta-analytic reviews involving a wide variety of internalizing problems and treatment trials focused specifically on youth depression in the current review. First, it may be true that evidence of differential effectiveness is based primarily on a different subset of internalizing problems, namely anxietybased conditions (e.g., phobias, generalized anxiety). As mentioned previously, none of the internalizing problem types in the Weisz et al. (1987) study were described as "depression" treatments. Further, only 6 of the 40 internalizing studies were coded as "depression" studies in the meta-analysis by Weisz et al. (1995), whereas in the remaining 32 internalizing studies, the identified problems were phobias/anxiety, somatic complaints, and withdrawal. Thus, the source of the interaction found in the previous studies might exist primarily in these internalizing domains (predominantly anxiety), but not necessarily in depression studies in particular.

A second possible interpretation of the negligible differences in therapy outcome produced by professionals and graduate students might be attributable, 
in part, to the fact that the graduate students who delivered the treatments were being closely supervised by professionals. In fact, of the 19 studies reviewed, only 3 did not explicitly refer to the treatments as "manualized." So, it appears that using a treatment manual when delivering treatment to depressed children and adolescents is the norm, at least in the empirical studies reviewed here. Given that both groups produced impressive and roughly commensurate results, perhaps the data provide a tacit endorsement of professionally-led or tightly supervised treatment of depressed youth.

With respect to age, no statistically significant differences were found when treating children versus adolescents. This is discrepant from the findings reported by Weisz et al. (1995) as well as those described by Michael and Crowley (2002). A possible interpretation of this inconsistency in the literature is based on the difficulty in developing an operationalized definition of child versus adolescent studies. Indeed, in both of the aforementioned studies in which it was reported that interventions were more effective for adolescents than children; a false dichotomy was created whereby child studies were defined as having a mean age of 12 or younger and adolescent studies were characterized by a sample with a mean age of 13 or older due to limitations in the reported data from these studies. Thus, the authors cautioned in each case that it was not possible to make firm conclusions regarding the interaction between age and treatment outcome. Given these limitations in the data regarding age, future researchers should report findings based on age in order to further address this potentially important interaction. Although our study provides some interesting insights regarding the relationship between the level of therapist training and outcome, the conclusions from these data must be considered with a few caveats. First, the literature regarding the treatment of depressed youth is rapidly expanding and the current findings need to be considered within this context. Second, our ability to interpret the possible interactions between particular variables (level of training, age) was hampered by the ways in which such data are collected, reported and categorized.

So where does this leave us, and where do we go from here? We suggest that although these findings are far from definitive given the aforementioned limitations, they offer some intriguing information about understudied contributors to therapy outcome, especially with depressed youth. Indeed, as it was pointed out previously, the therapist has been a "neglected variable" in outcome studies (see Elkin, 1999; Garfield, 1997). However, in order to disentangle the main effects from possible moderators of outcome, we first need to do a better job of reporting the findings based upon therapist variables and elevate these often-secondary questions to primary hypotheses. This also assumes that there is sufficient variability in the characteristic under study to make it a meaningful analysis. Crits-Christoph and Mintz (1991) suggested that in order to consider how particular therapist variables might be associated with outcome, a sufficient number of therapists must be included to have reasonably high levels of statistical power. Moreover, it would be helpful to conduct entire studies where a more systematic analysis of therapist variables and their potential contribution to outcome are of primary import. 
In sum, these data provide some important insights about treating depressed youth. It seems reasonable to ask whether professional training enhances one's ability to conduct psychotherapy. While the current study does not find evidence for the superiority of professional therapists in the treatment of youth depression, it does not endorse the idea that graduate student therapists may be more cost effective alternatives to obtain equal clinical effects. Instead, we offer the suggestion that graduate students are trained and deliver therapy within a rich, multi-layered supervisory team, which often includes professionals, manualized interventions, and tightly supervised treatment protocols. Indeed, Crits-Christoph et al. (1991) have demonstrated that the use of treatment manuals reduces the variability associated with therapist characteristics. Thus, we should not be surprised that graduate students in these contexts produce results commensurate with professionals.

\section{References}

*References Marked with an asterisk indicate studies included in the meta-analysis.

Achenbach, T. M., \& McConaughy, S. H. (1992). Taxonomy of internalizing disorders of childhood and adolescence. In W. M. Reynolds (Ed.), Internalizing disorders in children and adolescents (pp. 19-60). New York: Wiley.

Becker, B. J. (1988). Synthesizing standardized mean-change measures. British Journal of Mathematical and Statistical Psychology, 41, 257-278.

Berman, J. S., \& Norton, N. C. (1985). Does professional training make a therapist more effective? Psychological Bulletin, 98, 401-406.

Beutler, L. E., Machado, P. P. P., \& Allstetter Neufeldt, S. (1994). Therapist variables. In A. E. Bergin \& S. L. Garfield (Eds.), Handbook of psychotherapy and behavior change (3rd ed., pp. 229-269). New York: Wiley.

*Brent, D. A., Holder, D., Kolko, D., Birmaher, B., Baugher, M., Roth, C., Iyengar, S., \& Johnson, B. A. (1997). A clinical psychotherapy trial for adolescent depression comparing cognitive, family, and supportive therapy. Archives of General Psychiatry, 54, 877-885.

*Butler, L., Miezitis, S., Friedman, R., \& Cole, E. (1980). The effect of two school-based intervention programs on depressive symptoms in preadolescents. American Education Research Journal, 17, 111-119.

Clarke, G. N., Rohde, P., Lewinsohn, P. M., Hops, H., \& Seeley, J. R. (1999). Cognitivebehavioral treatment of adolescent depression: Efficacy of acute group treatment and booster sessions. Journal of the American Academy of Child and Adolescent Psychiatry, 38, 272-279. 
Cohen, J. (1960). A coefficient of agreement for nominal scales. Educational and Psychological Measurement, 20, 37-46.

Cohen, J. (1988). Statistical power analysis for the behavioral sciences (2nd ed.). Hillsdale, NJ: Elrbaum.

Christensen, A., \& Jacobson, N. S. (1994). Who (or what) can do psychotherapy: The status and challenge of nonprofessional therapies. Psychological Science, 5, 8-14.

Crits-Christoph, P., Baranackie, K., Kurcias, J. S., Beck, A. T., Carroll, K., Perry, K., et al. (1991). Meta-analysis of therapist effects in psychotherapy outcome studies. Psychotherapy Research, 1, 81-91.

Crits-Christoph, P., \& Mintz, J. (1991). Implications of therapist effects for the design and analysis of comparative studies of psychotherapies. Journal of Consulting and Clinical Psychology, 59, 20-26.

*Curry, J. F., \& Wells, K. C. (1998, August). Treating comorbid depression and substance abuse in adolescents. Paper presented at the Annual Convention of the American Psychological Association, San Francisco, CA.

Curry, J. F., Wells, K. C., Lochman, J. E., Craighead, W. E., \& Nagy, P. D. (2003). Cognitivebehavioral intervention for depressed, substance abusing adolescents: Development and pilot testing. Journal of the American Academy of Child and Adolescent Psychiatry, 42, 656-665.

DuPaul, G. J., \& Eckert, T. L. (1997). The effects of school-based interventions for attentiondeficit hyperactivity disorder: A meta-analysis. School Psychology Review, 26, 5-27.

Durlak, J. (1979). Comparative effectiveness of paraprofessional and professional helpers. Psychological Bulletin, 86, 80-92.

Elkin, I. (1999). A major dilemma in psychotherapy outcome research: Disentangling therapists from therapies. Clinical Psychology: Science and Practice, 6, 10-32.

*Fine, S., Forth, A., Gilbert, M., \& Haley, G. (1991). Group therapy for adolescent depressive disorder: A comparison of social skills and therapeutic support. Journal of the American Academy of Child and Adolescent Psychiatry, 30, 79-85.

Garcia, J. A., \& Weisz, J. R. (2002). When youth mental care stops: Therapeutic relationship problems and other reasons for ending youth outpatient treatment. Journal of Consulting and Clinical Psychology, 70, 439-443.

Garfield, S. L. (1997). The therapist as a neglected variable in psychotherapy research. Clinical Psychology: Science and Practice, 4, 40-43. 
Glass, G. V. (1977). Integrating findings: The meta-analysis of research. Review of Research in Education, 5, 351-379.

Hattie, J. A., Sharpley, C. F., \& Rogers, H. J. (1984). Comparative effectiveness of professional and paraprofessional helpers. Psychological Bulletin, 95, 534-541.

Hedges, L. V., \& Olkin, I. (1985). Statistical methods for meta-analysis. Orlando, FL: Academic Press.

*Hickman, K. A. (1994). Effects of social skills training on depressed children attending a behavioral day treatment program. Unpublished doctoral dissertation. Hofstra University, New York, NY.

Johnson, B. T. (1989). DSTAT: Softward for the meta-analytic review of research literatures. Hillsdale, NJ: Erlbaum.

*Kahn, J. S., Kehle, T. J., Jenson, W. R., \& Clark, E. (1990). Comparison of cognitivebehavioral, relaxation, and self-modeling interventions for depression among middle-school students. School Psychology Review, 19, 196-211.

Kolko, D. J., Brent, D. A., Baugher, M., Bridge, J., \& Birmaher, B. (2000). Cognitive and family therapies for adolescent depression: Treatment specificity, mediation, and moderation. Journal of Consulting and Clinical Psychology, 68, 603-614.

*Lewinsohn, P. M., Clarke, G. N., Hops, H., \& Andrews, J. (1990). Cognitive-behavioral treatment of depressed adolescents. Behavior Therapy, 21, 385-401.

*Lewinsohn, P. M., Clarke, G. N., Rohde, P., Hops, H., \& Seeley, J. (1996). A course in coping: A cognitive-behavioral approach to the treatment of adolescent depression. In E. D. Hibbs \& P. S. Jensen (Eds.), Psychosocial treatments for child and adolescent disorders: Empirically-based strategies for clinical practice (pp. 109-135). Washington, DC: American Psychological Association.

*Liddle, B., \& Spence, S. H. (1990). Cognitive-behavioral therapy with depressed primary school children: A cautionary note. Behavioral Psychotherapy, 18, 85-102.

Lipsey, M. W., \& Wilson, D. B. (2001). Practical meta-analysis. Thousand Oaks, CA: Sage.

Michael, K. D., \& Crowley, S. L. (2002). How effective are treatments for child and adolescent depression: A meta-analytic review. Clinical Psychology Review, 22, 247-269.

*Mufson, L., Moreau, D., Weissman, M. M., Wickramaratne, P., Martin, J., \& Samoilov, A. (1994). Modification of interpersonal psychotherapy with depressed adolescents (IPT-A): Phase I and II studies. Journal of the American Academy of Child and Adolescent Psychiatry, 33, 695705 . 
*Mufson, L., Weissman, M. M., Moreau, D., \& Garfinkel, R. (1999). Efficacy of interpersonal psychotherapy for depressed adolescents. Archives of General Psychiatry, 56, 573-579.

Patterson, C. H. (1984). Empathy, warmth, genuineness in psychotherapy: A review of reviews. Psychotherapy, 21, 431-438.

*Reed, M. K. (1994). Social skills training to reduce depression in adolescents. Adolescence, 29, 293-302.

Reinecke, M. A., Ryan, M. A., \& DuBois, D. L. (1998). Cognitive-behavioral therapy of depression and depressive symptoms during adolescence: A review and meta-analysis. Journal of the American Academy of Child and Adolescent Psychiatry, 37, 26-34.

Reynolds, W. M. (1990). Introduction to the nature and study of internalizing disorders in children and adolescents. School Psychology Review, 2, 137-141.

*Reynolds, W. M., \& Coats, K. I. (1986). A comparison of cognitive-behavioral therapy and relaxation training for the treatment of depression and adolescents. Journal of Consulting and Clinical Psychology, 54, 653-660.

Rosenthal, R. (1979). The "file drawer problem" and tolerance for null results. Psychological Bulletin, 86, 638-641.

Rosenthal, R. (1994). Parametric measures of effect size. In H. Cooper \& L. V. Hedges (Eds.), The handbook of research synthesis. New York: Russell Sage Foundation.

*Rosselló, J., \& Bernal, G. (1999). The efficacy of cognitive-behavioral and interpersonal treatments for depression in Puerto Rican Adolescents. Journal of Consulting and Clinical Psychology, 67, 734-745.

*Santor, D. A., \& Kusumakar, V. (2001). Open trial of interpersonal therapy in adolescents with moderate to severe major depression: Effectiveness of novice IPT therapists. Journal of the American Academy of Child and Adolescent Psychiatry, 40, 236-240.

*Stark, K. D., Reynolds, W. M., \& Kaslow, N. J. (1987). A comparison of the relative efficacy of self-control therapy and a behavioral problem-solving therapy for depression in children. Journal of Abnormal Child Psychology, 15, 91-113.

Stein, D. M., \& Lambert, M. J. (1984). On the relationship between therapist experience and outcome. Clinical Psychology Review, 4, 127-142.

Stein, D. M., \& Lambert, M. J. (1995). Graduate training in psychotherapy: Are therapy outcomes enhanced? Journal of Consulting and Clinical Psychology, 63, 182-196. 
*Vostanis, P., Feehan, C., Grattan, E., \& Bickerton, W. L. (1996). A randomised controlled outpatient trial of cognitive-behavioural treatment for children and adolescents with depression: 9-month follow-up. Journal of Affective Disorders, 40, 105-116.

*Weisz, J. R., Thurber, C. A., Sweeney, L., Proffitt, V. D., \& LeGagnoux, G. L. (1997). Brief treatment of mild-to-moderate child depression using primary and secondary control enhancement training. Journal of Consulting and Clinical Psychology, 65, 703-707.

Weisz, J. R., Weiss, B., Alicke, M. D., \& Klotz, M. L. (1987). Effectiveness of psychotherapy with children and adolescents: A meta-analysis for clinicians. Journal of Consulting and Clinical Psychology, 55, 542-549.

Weisz, J. R., Weiss, B., Han, S. S., Granger, D. A., \& Morton, T. (1995). Effects of psychotherapy with children and adolescents revisited: A meta-analysis of treatment outcome studies. Psychological Bulletin, 117, 450-468.

Wilson, S. J., Lipsey, M. W., \& Derzon, J. H.(2003). The effects of school-based intervention programs on aggressive behavior: A meta-analysis. Journal of Consulting and Clinical Psychology, 71, 136-149.

*Wood, A., Harrington, R., \& Moore, A. (1996). Controlled trial of a brief cognitive-behavioral intervention in adolescent patients with depressive disorders. Journal of Child Psychology and Psychiatry, 37, 737-746. 